\title{
ON MAXIMALITY OF GORENSTEIN SEQUENCES
}

\author{
MARIA GRAZIA MARINARI ${ }^{1}$
}

\begin{abstract}
It is well known that if $A$ is a Gorenstein ring, then every ideal generated by a regular sequence $\mathbf{x} \subset A$ has irreducible (minimal) primary components. This feature led us to define a Gorenstein sequence of a ring $A$ to be any ordered regular sequence $\mathbf{x}=\left\{x_{1}, \ldots, x_{r}\right\} \subset A$ such that for every $i \in\{1, \ldots, r\}$ the ideal $\left(x_{1}, \ldots, x_{i}\right)$ has irreducible minimal primary components. We showed for Gorenstein sequences (G-sequences for short) some parallels of well-known properties of regular sequences and moreover by means of $\mathbf{G}$-sequences we gave the following natural characterization of local Gorenstein rings: "A local ring $(A, \mathrm{~m})$ is Gorenstein iff $\mathrm{m}$ contains a $\mathbf{G}$ sequence of length $=K-\operatorname{dim} A$ ".

In this note we are going to give some information about "maximality" of G-sequences in a local ring $A$, producing sufficient conditions on $A$ in order that the maximal G-sequences of $A$ all have the same length, i.e. in order to give a "good" definition of G-depth $A$. Furthermore, we will state some results about the G-depth behavior with respect to local flat ring homomorphisms.
\end{abstract}

0 . Notations and preliminary results. Throughout this paper $A$ will denote a commutative noetherian ring with unity. In addition, we will say that the Gorenstein locus of $A$ is open if there exists an ideal $\mathfrak{\Im} \subset A$ such that for every $\mathfrak{p} \in \operatorname{Spec} A, A_{\mathfrak{p}}$ is a local Gorenstein ring iff $\mathfrak{p} D \mathfrak{s}$. In [H. K., Vortrag 6] it has been shown that the Gorenstein locus of a local Cohen-Macaulay (C. M. for short) ring $A$ is open if there exists the canonical module $K_{A}$ (for the definition and properties of $K_{A}$ see [H. K., Vortrag 5] where it has also been given a sufficient criterion for the existence of $K_{A}$ ). If $K_{A}$ exists then the ideal

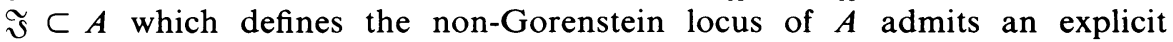
description (see [H.K., Bemerkung and Lemma 6.19]) as the ideal generated by the elements $\varphi(x)$ with $x \in K_{A}, \varphi \in \operatorname{Hom}_{A}\left(K_{A}, A\right)$, i.e. $\mathfrak{\Im}$ is the trace ideal of $K_{A}$ (cf. [De M. I., Chapter 1, §1B]).

In this connection we have the following handy characterization:

0.1 . If $A$ is a ring with open Gorenstein locus and $\mathfrak{} \subset A$ is the ideal defining the non-Gorenstein locus of $A$, then an ordered regular sequence $\left\{x_{1}, \ldots, x_{r}\right\} \subset A$ is a $\mathbf{G}$-sequence iff for every $i \in\{1, \ldots, r\}$ and $p$ $\in \operatorname{Ass}\left(A /\left(x_{1}, \ldots, x_{i}\right)\right)$ we have $\mathfrak{p} D \mathfrak{s}$.

Now we recall explicitly two concepts which will be frequently used in the following.

Received by the editors April 21, 1975.

AMS (MOS) subject classifications (1970). Primary 13H10; Secondary 18H20.

Key words and phrases. Commutative noetherian Cohen-Macaulay Gorenstein ring, Gorenstein sequence, canonical module.

${ }^{1}$ Supported by a scholarship of the Italian Council of Research. 
0.2. The $\mathbf{G}$-depth of a local ring $(A, \mathrm{~m})$ is the largest number of elements in m making up a $\mathbf{G}$-sequence of $A$. If $\mathrm{m}$ does not contain any $\mathbf{G}$-sequence of positive length, we define the $\mathbf{G}$-depth $A$ to be 0 if the zero ideal of $A$ has irreducible minimal primary components (i.e. the empty set $\varnothing \subset A$ makes up a $\mathbf{G}$-sequence of length 0 ), otherwise $\mathbf{G}$-depth $A=-\infty$ (cf. [ $\mathbf{M}_{1}$, Definition 4.1]).

0.3. A ring $A$ is said to be $\mathbf{G}_{n}$ if $\mathbf{G}$-depth $A_{\mathfrak{p}} \geqslant \min (n$, ht $\mathfrak{p})$ for every $\mathfrak{p} \in \operatorname{Spec} A$ (cf. [ $\mathbf{M}_{1}$, Definition 4.5.]).

In $\left[\mathbf{M}_{\mathbf{2}}\right.$, Theorem 2.1] we gave several equivalent characterizations for the $\mathbf{G}_{n}$ condition, showing in particular that a $\operatorname{ring} A$ is $\mathbf{G}_{n}$ iff it is $S_{n}$ (i.e. depth $A_{\mathfrak{p}} \geqslant \min (n, \mathrm{ht} \mathfrak{p})$ for every $\left.\mathfrak{p} \in \operatorname{Spec} A\right)$ and $A_{\mathfrak{p}}$ is a local Gorenstein ring for every $\mathfrak{p} \in \operatorname{Spec} A$ such that ht $\mathfrak{p} \leqslant n$. Here we can add the following:

0.4. If $A$ is an $S_{n}$ ring with open Gorenstein locus and $\mathfrak{\Im} \subset A$ is the ideal defining the non-Gorenstein locus of $A$, then $A$ is $\mathbf{G}_{n}$ iff ht $\mathfrak{\Im}>n$.

\section{Principal results and examples.}

Proposition 1.1 . Let $A$ be any local C. M. ring with open Gorenstein locus and let $\mathfrak{s} \subset A$ be the ideal which defines the non-Gorenstein locus of $A$; then:

(a) if ht $\mathfrak{\Im}=0, A$ does not contain any $\mathbf{G}$-sequence;

(b) if ht $\mathfrak{\Im}>0$, all maximal G-sequences of $A$ have the same length (exactly equal to $K-\operatorname{dim} A$ or $K-\operatorname{dim} A-1$, according as $A$ is Gorenstein or not).

Proof. (a) $A$ is not a $\mathbf{G}_{0}$ ring (cf. 0.4 ) so it is clear that $A$ does not contain any $\mathbf{G}$-sequence of length $=0$ (cf. [ $\mathbf{M}_{2}$, Osservazione (iii)]). On the other hand one can easily see that $A$ does not even contain $\mathbf{G}$-sequences of positive length since if there existed $\mathbf{x} \subset A$ a $\mathbf{G}$-sequence of length $=r>0$, then both $A /(\mathbf{x})$ and $A$ would be $\mathbf{G}_{0}$ rings (respectively by $\left[\mathbf{M}_{\mathbf{2}}\right.$, Osservazione (iv)] and $\left[\mathbf{M}_{\mathbf{3}}\right.$, Lemma 2.2 ]) and this would clearly give a contradiction.

(b) First of all we observe that if $A$ is a Gorenstein ring, then manifestly all maximal $\mathbf{G}$-sequences of $A$ must have the same length exactly equal to $K-\operatorname{dim} A$ since it follows directly from the definitions that all regular sequences of any (not necessarily local) Gorenstein ring are also G-sequences (cf. [B, Fundamental Theorem], and [ $\mathbf{M}_{1}$, Definition 2.1]). Therefore to complete our proof we have only to examine the case $\mathfrak{s} \varsubsetneqq A$. Here ht $\mathfrak{s}>0$ implies that for every $\mathfrak{p} \in \operatorname{Spec} A$ such that ht $\mathfrak{p}=0, A_{\mathfrak{p}}$ is a local Gorenstein ring (cf. 0.4): hence all (minimal) primary components of the zero ideal in $A$ are irreducible, i.e. the empty set $\varnothing \subset A$ is a $\mathbf{G}$-sequence of length $=0$ (cf. $\left[\mathbf{M}_{2}\right.$, Osservazione (vi)]). This just proves our theorem in case $K-\operatorname{dim} A=1$ where $\varnothing$ is actually the only (maximal) $\mathbf{G}$-sequence of $A$ (we are assuming $A$ is not Gorenstein), so from now on we can suppose $K-\operatorname{dim} A>1$. Since $A$ is a C. M. ring ht $\mathfrak{\Im}>0$ implies also that there exists some element $i \in$ is which is regular in $A$ and so can be completed to a maximal regular sequence of $A$, say $\mathbf{x}=\left\{i, x_{2}, \ldots, x_{m}\right\}$ with $m=K-\operatorname{dim} A$. We want to show that $\mathbf{x}^{\prime}=\left\{x_{2}, \ldots, x_{m}\right\}$ is a $\mathbf{G}$-sequence of $A$; in this connection to say that $\mathbf{x} \subset A$ is a regular sequence means in particular that $i \notin \mathfrak{P}^{\prime}$ for any $\mathfrak{P}^{\prime}$ $\in \operatorname{Ass}\left(A /\left(\mathbf{x}^{\prime}\right)\right)$, i.e. $A_{\mathfrak{P}^{\prime}}$ is a local Gorenstein ring for every $\mathfrak{B}^{\prime} \in$ Ass $\left(A /\left(\mathbf{x}^{\prime}\right)\right)$ and this means that $\mathbf{x}^{\prime}$ is a $\mathbf{G}$-sequence, so $\varnothing$ is not a maximal $\mathbf{G}$-sequence in $A$. Then let $\mathbf{y} \subset A$ be any maximal $\mathbf{G}$-sequence of positive length $=s<m$ 
(recall that we are assuming $A$ is not Gorenstein): for every $\mathfrak{A} \in \operatorname{Ass}(A /(\mathbf{y}))$ clearly $\mathfrak{D} \mathfrak{\Im}$ so there exists some element $j \in \mathfrak{\exists}$ which is a non-zero-divisor modulo (y) such that $\{\mathbf{y}, j\}$ is a regular sequence of length $=s+1$. If $s<m-1,\{\mathbf{y}, j\}$ can be completed to a maximal regular sequence of $A$, say $\left\{\mathbf{y}, j, y_{s+2}, \ldots, y_{m}\right\}$; here applying the same argument as above we can see that $\left\{\mathbf{y}, y_{s+2}, \ldots, y_{m}\right\}$ is a $\mathbf{G}$-sequence of $A$ containing $\mathbf{y}$, contradicting the maximality of $\mathbf{y}$, so all maximal $\mathbf{G}$-sequences of $A$ actually have length $=K-\operatorname{dim} A$ -1 .

Corollary 1.2. If $A$ is a local $C$. M. ring with open Gorenstein locus, then G-depth $A$ is the length of any maximal G-sequence in $A$ (as usual G-depth $A$ $=-\infty$ if $A$ does not contain $\mathbf{G}$-sequences of any length).

Corollary 1.3. Let $A$ be any $S_{n}$ local ring with $K-\operatorname{dim} A>n>1$. If the Gorenstein locus of $A$ is open, then either $A$ does not contain any G-sequence or all maximal G-sequences of $A$ have length $\geqslant n-1$.

Proof. Let $\mathfrak{\Im}$ be the ideal which defines the non-Gorenstein locus of $A$; we can observe that, as in Proposition 1.1, our conclusion and proof depend on height $\mathfrak{\Im}$. Precisely: ht $\mathfrak{\Im}=0$ implies both $A$ does not contain any G-sequence of length $=0$ (in fact in this case $A$ is not $\mathbf{G}_{0}$ ) and $A$ does not contain any $\mathbf{G}$ sequence of positive length (namely if $\mathbf{x}=\left\{x_{1}, \ldots, x_{r}\right\}$ would be a $\mathbf{G}$-sequence of length $=r>0$, then for all $i \in\{1, \ldots, r\} x_{i}$ would be a $\mathbf{G}$-sequence which generates an unmixed ideal (cf. [S, Theorem 2.2]), so $A /\left(x_{i}\right)$ and then $A$ (by [R. F., Proposition 3]) would be $S_{1}$ and $\mathbf{G}_{0}$ rings contradicting the fact that $A$ is not $\mathbf{G}_{0}$ ).

ht $\mathfrak{\Im}>0$ implies $\varnothing$ is not a maximal G-sequence (we can use an argument like that of Proposition 1.1). Then let $\mathbf{x} \subset A$ be any maximal $\mathbf{G}$-sequence of length $=s>0$, if $s<n-1$ for every $\mathfrak{P} \in \operatorname{Ass}(A /(\mathbf{x})), \mathfrak{P} \nsubseteq \mathfrak{s},(\mathbf{x})$ is an unmixed ideal by [S, Theorem 2.2], so there exists some element $j \in \mathfrak{\Im}$ which is a non-zero-divisor modulo ( $\mathbf{x})$. Considering the regular sequence $\{\mathbf{x}, j\}$, we can conclude, as in Proposition 1.1:

Remark I. From [ $\mathbf{M}_{\mathbf{3}}$, Lemma 2.2] and [R. F., Proposition 3], we can deduce some information about the existence of $\mathbf{G}$-sequences in a local ring $A$ without having resort to the hypothesis that the Gorenstein locus of $A$ is open; precisely we can say:

(i) In any local non- $\mathbf{G}_{0}$ ring $A$ which satisfies the "saturated chain condition on prime ideals", there exist no G-sequences.

(ii) In any local non- $\mathbf{G}_{0}$ ring $A$ there exist no $\mathbf{G}$-sequences (of any length) which generated unmixed ideals, but a priori we do not have any information about possible G-sequences which generated mixed ideals.

REMARK II. From the proof of Corollary 1.3 we can deduce that in a local $S_{n} \operatorname{ring} A(K-\operatorname{dim} A>n \geqslant 1)$ which is $\mathbf{G}_{0}$ and has open Gorenstein locus, there exist $\mathbf{G}$-sequences of length $=\operatorname{depth} A-1$ but a priori we cannot say if this must be the length of every maximal $\mathbf{G}$-sequence of $A$, so actually we do not know whether there may exist maximal G-sequences of different lengths in $A$.

Recall explicitly the following notation introduced in [W.I.T.O., Definition 1.7 ]. 
Definition 1.4. A ring homomorphism $\varphi: A \rightarrow B$ is Gorenstein if it is flat and has Gorenstein fibres.

Lemma 1.5. Let $A$ be any ring with open Gorenstein locus. Then, for every Gorenstein homomorphism $\varphi: A \rightarrow B$, the Gorenstein locus of $B$ is open.

Proof. Let $f: Y=\operatorname{Spec} B \rightarrow \operatorname{Spec} A=X$ be the induced morphism and let $U \subset X$ be the Gorenstein locus of $A$. For every $\mathfrak{P} \in f^{-1}(U), B_{\mathfrak{B}}$ is Gorenstein; namely, putting $\mathfrak{p}=f(\mathfrak{B})$, clearly $\mathfrak{p} \in U$ so the local homomorphism $\psi: A_{\mathfrak{p}} \rightarrow B_{\mathfrak{p}}$ (induced by $\varphi$ ) is flat with $A_{\mathfrak{p}}$ Gorenstein (since $\mathfrak{p} \in U$ ) and $B_{\mathfrak{P}} / \mathfrak{p} B_{\mathfrak{P}}$ Gorenstein (since it is a localization of the fibre of $\varphi$ at $\mathfrak{p}$ ). On the other hand, for every $\mathfrak{Q} \in Y-f^{-1}(U), B_{\mathfrak{Q}}$ is not Gorenstein since putting $\mathfrak{q}=\mathfrak{\Omega} \cap A$ clearly $\mathfrak{a} \notin U$, so $A_{\mathfrak{q}}$ is not Gorenstein. Therefore the Gorenstein locus of $B$ is precisely $f^{-1}(U)$ which, by the hypothesis, is clearly open.

Proposition 1.6. Let $(A, \mathrm{~m})$ be any local $C$. M. ring with open Gorenstein locus. Then for every local Gorenstein homomorphism $\varphi: A \rightarrow B$, we have

$$
\mathbf{G}-\operatorname{depth} B=\mathbf{G}-\operatorname{depth} A+\mathbf{G}-\operatorname{depth} B / \mathrm{m} B \text {. }
$$

Proof. Observe that under the given hypotheses not only $B$ is a (local) C. M. ring (cf. [D, Corollary 5.1]) but also its Gorenstein locus is open (cf. Lemma 1.5 ): therefore $\mathbf{G - d e p t h ~} B$ is actually well defined (cf. Corollary 1.2 ). In addition, we notice that if $A$ is Gorenstein, then there is nothing to prove, since in that case $B$ is a (local) Gorenstein ring by [W.I.T.O., Theorem 1], so clearly

$$
\begin{aligned}
\mathbf{G}-\operatorname{depth} B & =K-\operatorname{dim} B=K-\operatorname{dim} A+K-\operatorname{dim} B / \mathrm{m} B \\
& =\mathbf{G}-\operatorname{depth} A+\mathbf{G}-\operatorname{depth} B / \mathrm{m} B .
\end{aligned}
$$

Then, to show our contention, we only have to examine the case $A$ is not Gorenstein which, according to Proposition 1.1, splits into $\mathbf{G}-\operatorname{depth} A$ $=-\infty$ and $\mathbf{G}-\operatorname{depth} A=K-\operatorname{dim} A-1 \quad$ (automatically $\geqslant 0$ ). If Gdepth $A=-\infty$, then we cannot have $\mathbf{G}-\operatorname{depth} B \geqslant 0$, since that would mean $B$ is at least $\mathbf{G}_{0}$ and hence also $A$ would be at least $\mathbf{G}_{0}$ (cf. [ $\mathbf{M}_{1}$, Theorem 5.1]), contradicting $\mathbf{G}$ - $\operatorname{depth} A=-\infty$; then

$$
\begin{aligned}
\mathbf{G}-\operatorname{depth} B & =-\infty=-\infty+K-\operatorname{dim} B / \mathrm{m} B \\
& =\mathbf{G}-\operatorname{depth} A+\mathbf{G}-\operatorname{depth} B / \mathrm{m} B .
\end{aligned}
$$

If $0 \leqslant \mathbf{G}-\operatorname{depth} A=K-\operatorname{dim} A-1$, then $B$ is a $\mathbf{G}_{0}$ ring (cf. [ $\mathbf{M}_{\mathbf{1}}$, Corollario 5.2]), that is $\mathbf{G}$ - depth $B \geqslant 0$. Moreover, since we are assuming $A$ is not Gorenstein, we cannot have $\mathbf{G}-\operatorname{depth} B=K-\operatorname{dim} B$ (cf. [W.I.T.O., Theorem 1]); thus again

$$
\begin{aligned}
\mathbf{G}-\operatorname{depth} B & =K-\operatorname{dim} B-1=K-\operatorname{dim} A-1+K-\operatorname{dim} B / \mathrm{m} B \\
& =\mathbf{G}-\operatorname{depth} A+\mathbf{G}-\operatorname{depth} B / \mathrm{m} B .
\end{aligned}
$$

REMARK III. Observe that if the local C. M. ring $(A, \mathrm{~m})$ has the canonical module $K_{A}$, then every local flat ring homomorphism $\varphi: A \rightarrow B$ such that 
$B / \mathrm{m} B$ is Gorenstein (i.e. $K_{B} \simeq K_{A} \otimes_{A} B$ (cf. [H.K., Satz 6.14.])) is actually a Gorenstein homomorphism. Namely, using the same notations as in Lemma 1.5, for any $\mathfrak{p} \in X$, the fibre of $\varphi$ at $\mathfrak{p}$ is Gorenstein since (cf. [E.G.A., $\mathrm{IV}_{2}$, Lemma 7.3.2]) for every $\mathfrak{P} \in f^{-1}(\mathfrak{p}), B_{\mathfrak{P}} / \mathfrak{p} B_{\mathfrak{P}}$ is Gorenstein, being

$$
\begin{aligned}
\left(K_{A_{\mathfrak{p}}}\right) \otimes_{A_{\mathfrak{p}}} B_{\mathfrak{P}} & =\left(K_{A}\right)_{\mathfrak{p}} \otimes_{A_{\mathfrak{p}}} B_{\mathfrak{P}}=K_{A} \otimes_{A} A_{\mathfrak{p}} \otimes_{A_{\mathfrak{p}}} B_{\mathfrak{P}}=K_{A} \otimes_{A} B_{\mathfrak{P}} \\
& =K_{A} \otimes_{A} B \otimes_{B} B_{\mathfrak{P}}=K_{B} \otimes_{B} B_{\mathfrak{B}}=\left(K_{B}\right)_{\mathfrak{P}}=K_{B_{\mathfrak{P}}}
\end{aligned}
$$

(cf. [R, (3) Theorem] and [H.K., Korollar 5.25]). Notice that the hypothesis " $B / \mathrm{m} B$ is Gorenstein" cannot be avoided; namely, we can easily see that Proposition 1.6 does not hold for a local flat homomorphism $\varphi: A \rightarrow B$ of complete, equidimensional local rings such that $A$ is Gorenstein and $B$ is $\mathbf{G}_{0}$ but not Gorenstein (e.g., fix a field $k$, take

$$
\begin{aligned}
& A=k\left[\left[U^{N}\right]\right] \quad(N=1 . c . m .(6,7,8,9)), \\
& B=k[[x, y, z, t]] /\left(t^{2}-x^{3}, z^{2}-y t, y^{2}-x z, y z-x t\right),
\end{aligned}
$$

and $\varphi$ the inclusion map (which is clearly flat and local); what we get is $\mathbf{G}$ - depth $A=1, \mathbf{G}$ - depth $B=0$ ( $B$ is a 1-dimensional complete integral domain which is not Gorenstein (cf. [K, Theorem]), and $\mathbf{G}-\operatorname{depth} B / \mathrm{m} B$ $=-\infty)$.

Finally we are going to list explicitly some examples of local flat ring homomorphisms for which Proposition 1.6 holds.

1.7. Let $(A, \mathrm{~m})$ be any local C.M. ring with residue field $k$ such that $K_{A}$ exists. Then:

(i) If $x$ is an indeterminate, for every maximal ideal $\mathfrak{M} \subset A[x]$ such that $\mathfrak{M} \cap A=\mathfrak{m}$, we have $\mathbf{G}-\operatorname{depth} A[x]_{\mathfrak{M}}=\mathbf{G}-\operatorname{depth} A+1$ (in fact the fibre $k \otimes_{A} A[x]_{\mathfrak{M}}$ is isomorphic to the 1-dimensional Gorenstein ring $k[x]$ localized at the maximal ideal $\tilde{\mathfrak{M}}=\mathfrak{M} k[x]$ (cf. [G.S., Example 12.1])).

(ii) If $F$ is a finite abelian group, for every maximal ideal $\mathfrak{M} \subset A[F]$, we have $\mathbf{G}$ - depth $A[F]_{\mathfrak{M}}=\mathbf{G}$ - $\operatorname{depth} A$ (in fact $k \otimes_{A} A[F]_{\mathfrak{M}}$ is isomorphic to the 0 -dimensional Gorenstein ring $k[F]$ localized at the maximal ideal $\overline{\mathfrak{M}}=\mathfrak{M k} k F](\mathrm{cf} .[\mathbf{P}$, Corollaire 2])).

(iii) If ${ }^{h}$ is the henselization of $A$ with respect to $m$, we have $\mathbf{G}-\operatorname{depth}{ }^{h} A$ $=\mathbf{G}-\operatorname{depth} A$ (in fact $k \otimes_{A}{ }^{h} A \simeq{ }^{h} A / \mathrm{m}^{h} A \simeq k$ (cf. [E.G.A., IV I $_{4}$, Theorem 18.6.6])).

(iv) If $\hat{A}$ is the $m$-adic completion of $A$, we have $\mathbf{G}-\operatorname{depth} \hat{A}=\mathbf{G}-\operatorname{depth} A$ (in fact $k \otimes_{A} \hat{A} \simeq \hat{A} / \mathrm{m} \hat{A} \simeq k$ (cf. [D, §6.A5])).

(v) If $A[[x]]$ is the formal power series ring (in one indeterminate) over $A$, we have $\mathbf{G}$ - depth $A[[x]]=\mathbf{G}$ - depth $A+1$ (in fact the fibres of $A \rightarrow A[[x]]$ are canonically isomorphic to the fibres of $B \rightarrow B[[x]]$ (where $B$ is a local Gorenstein ring such that $A=B / \mathfrak{b}$ (cf. [R, (3) Theorem])) at the prime ideals of $B$ containing $\mathfrak{b}$, and $B \rightarrow B[[x]]$ is a local flat ring homomorphism whose fibre at the closed point of $\operatorname{Spec} B$ is equal to $k \otimes_{B} B[[x]]$ which is clearly a (local) 1-dimensional Gorenstein ring (cf. [G.S., Theorem 9.8] and [W.I.T.O., Theorem 2])). 


\section{REFERENCES}

[B] H. Bass, On the ubiquity of Gorenstein rings, Math. Z. 82 (1963), 8-28. MR 27 \#3669.

[De M. I.] F. De Meyer and E. Ingraham, Separable algebras over commutative rings, Lecture Notes in Math., vol. 181, Springer-Verlag, Berlin and New York, 1971.

[D] J. Dieudonné, Topics in local algebra, Notre Dame Math. Lectures, no. 10, Univ. of Notre Dame Press, Notre Dame, Ind., 1967. MR 39 \#2748.

[E.G.A.] A. Grothendieck, Éléments de géométrie algébrique, Inst. Hautes Études Sci. Publ. Math. No. 24 (1965); ibid, No. 32 (1967). MR 33 \#330; 39 \#220.

[G. S.] S. Greco and P. Salmon, Topics in m-adic topologies, Ergebnisse der Mathematik und ihrer Grenzgebiete, Band 58, Springer-Verlag, Berlin and New York, 1971. MR 44 \# 190.

[H. K.] J. Herzog and E. Kunz, Der kanonische Modul eines C. M. Rings, Lecture Notes in Math., vol. 238, Springer-Verlag, Berlin and New York, 1971.

[K] E. Kunz, The value-semigroup of a one-dimensional Gorenstein ring, Proc. Amer. Math. Soc. 25 (1970), 748-751. MR 42 \#263.

[M $\left.\mathbf{M}_{1}\right]$ M. G. Marinari, Successioni di Gorenstein e proprietà $\mathbf{G}_{n}$, Rend. Sem. Mat. Univ. Padova 48 (1972).

$\left[\mathbf{M}_{2}\right]-$ Alcune carat terizzazioni degli anelli $\mathbf{G}_{n}$, Matematiche (Catania) 28 (1973).

$\left[\mathbf{M}_{3}\right]-$, Gorenstein sequences and $\mathbf{G}_{n}$ condition, J. Algebra (to appear).

[P] M. Paugam, La condition $\left(G_{q}\right)$ de Ischebeck, C. R. Acad. Sci. Paris Sér. A-B 276 (1973), A109-A112. MR 49 \#10683.

[R. F.] I. Reiten and R. Fossum, Commutative n-Gorenstein rings, Math. Scand. 31 (1972).

[R] I. Reiten, The converse to a theorem of Sharp on Gorenstein modules, Proc. Amer. Math. Soc. 32 (1972), 417-420. MR 45 \# 5128.

[S] R. Y. Sharp, Cousin complex characterizations of two classes of commutative Noetherian rings, J. London Math. Soc. (2) 3 (1971), 621-624. MR 45 \#3392.

[W.I.T.O.] K. Watanabe, et al., On tensor products of Gorenstein rings, J. Math. Kyoto Univ. 9 (1969), 413-423. MR 41 \#1716.

Italian Council of Research (C.N.R.), Piazzale delle Scienze 7, Rome, Italy

Current address: Istituto di Matematica, Università di Genova, 16132-via L. B. Alberti, 4, Genova, Italy 